\title{
Leishmania (Viannia) braziliensis growth in vitro culture relies more on folic acid availability than Leihsmania (Leishmania) amazonensis
}

\author{
Andrea Niño, Marcela Camacho*/+
}

\author{
Laboratorio de Biofísica, Centro Internacional de Física, Bogotá, Colombia *Departamento de Biología, Facultad de Ciencias, \\ Universidad Nacional de Colombia, Ciudad Universitaria, Calle 45, Carrera 30 Bogotá, Colombia
}

We compared the in vitro growth of promastigotes from two Leishmania species in TC-100 and Schneider media. Leishmania (Leishmania) amazonensis replication rates were similar in both tissue culture media and reached maximum rates by $48 \mathrm{~h}$. In contrast Leishmania (Viannia) braziliensis growth was significantly greater in TC-100 but maximum rates were achieved by 96 h. Folic acid appears to be the limiting factor and supplementation of Schneider media with this nutrient improved $\mathrm{L}$. (V.) braziliensis replication rates and decreased the time of maximum replication to $48 \mathrm{~h}$.

Key words: Leishmania (Viannia) braziliensis - Leishmania (Leishmania) amazonensis - replication rates - in vitro culture.

Leishmania are parasitic protozoa that infect many mammals including man. Despite the epidemiological impact of Leishmania (Viannia) braziliensis in the New World (OPS 1996), it has been studied less than other species, principally due to the difficulties of keeping this parasite in axenic conditions. These difficulties were used in the past to differentiate this Leishmania species and were related to media composition differences (Walton et al. 1977, Shaw \& Laison 1981). Biphasic media with solid phases enriched with variety of components have been used to keep Leishmania, but harvesting large numbers of parasites is difficult, thus liquid media is preferred for promastigote cultivation (Schuster \& Sullivan 2002). L. (V.) braziliensis promastigotes show low growth rates in in vitro cell culture, when they are compared with other Leishmania genus species (Armstrong \& Paterson 1994). In this study we compare the ability of two different media to sustain $L$. (V.) braziliensis and $L$. (L.) amazonensis growth.

\section{MATERIALS AND METHODS}

Parasites were cultured as previously described (Forero et al. 1999). L. (V.) braziliensis (HOM/BR/75M2903) and $L$. (L.) amazonensis (FLA/BR/67/PH8) isolates, kindly donated by Dr Nancy Gore Saravia (Cideim, Cali, Colombia) were used in the present study. Promastigotes, at an initial concentration of $1 \times 10^{6}$, were cultured at $24^{\circ} \mathrm{C}$ in 25 $\mathrm{cm}^{2}$ flasks (Costar) in TC-100 or Schneider media (Invitrogen) supplemented with 10\% FBS (Hyclone). Cultures were allowed to reach their metacyclic phase, deter-

Financial support: Colciencias, project 2228-04-12899, Programa Nacional de Ciencia y Tecnología de la Salud, División de Investigaciones project 90835, Universidad Nacional de Colombia, Centro Internacional de Física

${ }^{+}$Corresponding author. E-mail: mcamacho@ cable.net.co

Received 13 October 2004

Accepted 16 March 2005 mined by the stationary behavior of parasite number and rosette formation. Samples of parasites at different time points were counted in a Neubauer hematocytometer in a solution of 5\% Giemsa, $2 \%$ formaldehyde in $0.14 \mathrm{M} \mathrm{NaCl}$, under light microscopy. Data were analyzed with the Student's $t$ test and $p$ values $<0.05$ were considered significant.

\section{RESULTS}

L. (L.) amazonensis replication rates were similar in the two culture media during the period studied, and maximum replication rates occurred within $48 \mathrm{~h}$ (Fig. 1A). $L$. (V.) braziliensis replication rates doubled during the first $48 \mathrm{~h}$ and no differences were found between the two culture media $(\mathrm{p}=0.4)$. However, between 72 to $96 \mathrm{~h}$ postseeding, the replication rates of $L$. (V.) braziliensis promastigotes tripled in TC-100 but only doubled in Schneider. This difference was significant $(\mathrm{p}=0.02$; Fig. 1B). Although $L$. (V.) braziliensis took $96 \mathrm{~h}$ to reach its optimal replication rate compared to $L$. (L.) amazonensis, parasite numbers were similar. The percentage of metacyclics increased over the period studied for both species, and achieved their maximum value during the stationary phase. To further investigate the impact of particular nutritional factors on the recorded differences, folic acid was added to Schneider media. $L$. (V.) braziliensis replication rates increased and the maximum replication time was reduced to $48 \mathrm{~h}$ post-seeding, suggesting that the limiting nutritional factor was folic acid (Fig. 1C).

\section{DISCUSSION}

We found no differences between replication rates of $L$. (L.) amazonensis promastigotes in the two media studied. In contrast $L$. (V.) braziliensis promastigote replication rates were significantly higher in TC-100 compared to Schneider media. This difference may be the result of the presence of a toxic component in Schneider that is absent in TC-100 media, that $L$. (V.) braziliensis is more susceptible to. On the other hand, it could be the result of the depletion of an essential component in Schneider media that $L$. $(V$.) braziliensis needs. We rule out the first 

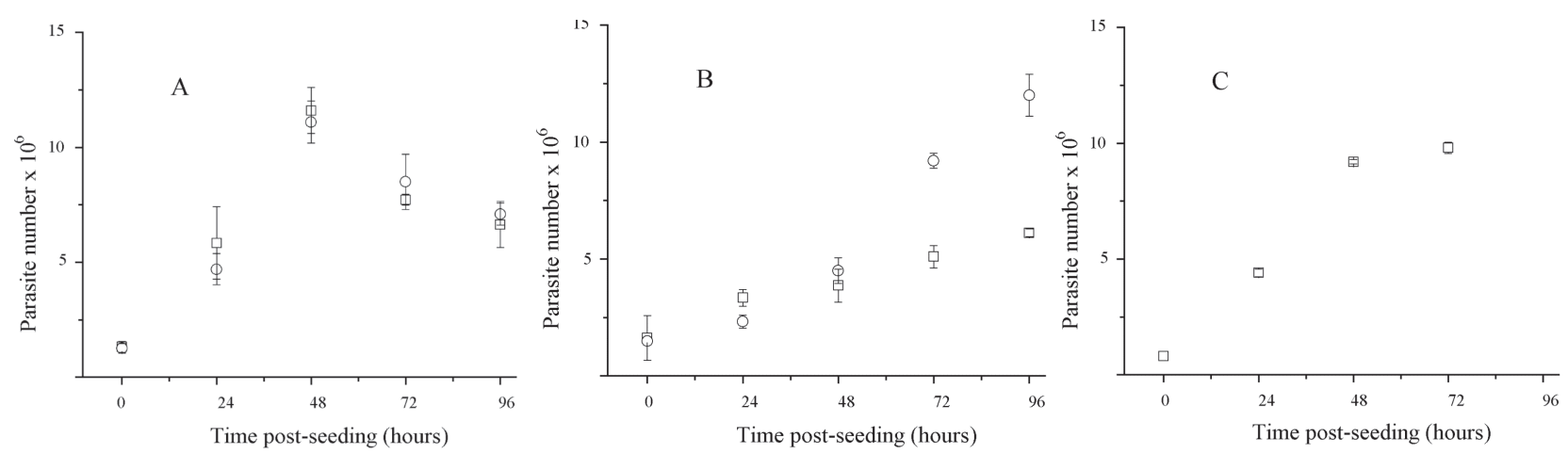

Fig. 1A: replication rates of Leishmania amazonensis promastigotes in $(\square)$ Schneider and (O) TC-100 media during 96 h; B: replication rates of L. braziliensis promastigotes in $(\square)$ Schneider and $(O)$ TC-100 media during $96 \mathrm{~h}$. For A and B data represents mean \pm SE of three independent experiments with three replicates each; C: replication rates of L. braziliensis promastigotes in ( $\square$ ) Schneider supplement with folic acid $0.02 \mathrm{mg} / \mathrm{l}$.

explanation because no differences were observed in the ability of these two media to sustain similar replication rates of this parasite during the first $48 \mathrm{~h}$ of culture. Nutrient requirements in Leishmania indicate that these parasites are folate and pterin auxotroph, but the requirements for these nutrients varies among Leishmania species. In $L$. (V.) braziliensis, this is particularly important since it has been reported that higher concentrations of folic acid are required by this species compared to other Leishmania (Steiger \& Steiger 1977). Folic acid has been implicated in tymidine and methionine synthesis and in the interconversion of serine into glycine by Leishmania (Ouellete et al. 2002). It was also showed that absence of folic acid inhibited $L$. (L.) donovani growth (Schuster \& Sullivan 2002). Therefore, we favor the depletion of an essential component as a more likely explanation. Schneider contains important amounts $(2 \mathrm{~g} / \mathrm{l})$ of a non defined component yeastolate, but lacks folic acid as a defined component. On the other hand, TC- 100 contains folic acid at micromolar concentrations (Gardiner \& Stockdale 1975), in the order or higher than those required by $L$. $(L$.$) donovani (Peixoto \& Beverley 1987$ ) and $L$. ( $L$.) major (Ellenberger \& Beverley 1987). Also, the fact that $L$. (V.) braziliensis replication rates improved and parasite growth was faster after the addition of folic acid to Schneider media adds further support to the hypothesis that depletion was responsible for the differences between the two media after $48 \mathrm{~h}$.

\section{REFERENCES}

Armstrong TC, Patterson JL 1994. Cultivation of Leishmania braziliensis in an economical serum-free medium containing human urine. J Parasitol 80: 1030-1032.
Ellenberger TE, Beverley SM 1987. Biochemistry and regulation of folate and methotrexate transport in Leishmania major. J Biol Chem 262: 10053-10058.

Forero ME, Marín M, Llano I, Moreno H, Camacho M 1999. Leishmania amazonensis infection induces changes in the electrophysiological properties of macrophage-like cells. J Membr Biol 170: 173-180.

Gardiner GR, Stockdale H 1975. Two tissue culture media for the production of lepidopteran cells and nuclear polihydrosis viruses. J Invertebr Pathol 25: 363-370.

OPS-Organización Panamericana de la Salud 1996. Epidemiología y Control de la Leishmaniasis en las Américas, por País o Territorio, Cuaderno técnico 44, Washington, 52 $\mathrm{pp}$.

Oullete M, Drummelsmitt J, Fadili AE, Künding C, Richard D, Roy G 2002. Pterin transport and metabolism in Leishmania and related trypanosomatids parasites. Int J Parasitol 32: 385-398.

Shaw JJ, Lainson R 1981. The in vitro cultivation of members of the Leishmania braziliensis complex. Trans $R$ Soc Trop Med Hyg 75: 127.

Schuster FL, Sullivan JJ 2002. Cultivation of clinically significant hemoflagellates. Clin Microbiol Rev 15: 374-389.

Peixoto MP, Beverley SM 1987. In vitro activity of sulfonamides and sulfones against Leishmania major promastigotes. Antimicrob Agents \& Chemother 31: 1575-1578.

Steiger RF, Steiger E 1977. Cultivation of Leishmania donovani and Leishmania braziliensis in defined media: nutritional requirements. J Protozool 24: 437-441.

Walton BC, Shaw JJ, Lainson R 1977. Observations on the in vitro cultivation of Leishmania braziliensis. J Parasitol 63: 1118-1119. 